\title{
Hot Gas Filtration and Heat Exchange in a Packed Bed Using Lapilli as a Granular Medium
}

\author{
Miguel Socorro, ${ }^{\dagger}$ Agustín Macías-Machín, ${ }^{\dagger}$ José M. Verona, ${ }^{\dagger}$ and Domingo Santana*,* \\ Energy and Environment Group (EMA), ETSII de Las Palmas, Edificio de Ingenierías, Campo Universitario \\ s/n, Tafira Baja, Universidad de Las Palmas de Gran Canaria, 35017 Las Palmas de Gran Canaria, Canary \\ Islands, Spain, and Energy Systems Engineering Group (ISE), Department of Thermal and Fluids Engineering, \\ Universidad Carlos III de Madrid, Avda. de la Universidad 30, 28911 Leganés, Madrid, Spain
}

A study was conducted on gas filtration and heat exchange in a fixed-bed heat exchanger-filter (FHEF) where we have removed fine dust particles from gases and exchanged heat with the granular medium (Lapilli). The influence of a number of variables was examined including gas velocities, gas temperatures, concentration of dust, height of the FHEF, dust and granular mean size, and pressure drop. The collection efficiency was found to decrease with increasing temperature, and the pressure drop increases when the size of the granular medium decreases. A numerical model for gas filtration and heat exchange using the finite element method was developed. It predicts the two-dimensional transient response of both solid and fluid phases as well as the variation of the specific deposit and void fraction of the FHEF with time. The numerical model incorporates variation in void fraction, specific surface area of the medium, and transport coefficient because of the combined processes of filtration and heat exchange.

\section{Introduction}

Granular bed filters are separators that utilize solids as a filter medium. Because of their relatively simple but robust construction, these separators have mainly been utilized as dust filters in different manufacturing industries.

The process of granular filtration is known for its relatively high collection efficiency for particles of widely ranging sizes and for its simplicity in operation. Other advantages of granular filtration include the abundance of granular substances, which can be used as filter grains, and the fact that many available granular substances are immune to high temperatures, high pressure, and/or chemically corrosive environments. ${ }^{1}$

Recently, we have proposed Lapilli as a new material in granular filtration, ${ }^{2}$ because Lapilli is used as a building material in the Canary Islands. It has the following uses: (a) as a material in the making of concrete, (b) as a granular material in the constructions of roads, and (c) as a hygroscopic material in gardens.

The Lapilli used in this paper is a volcanic slag characterized by variable versicularity, irregular morphology, and dark color. ${ }^{3}$ The Lapilli belongs to the basaltic rock family, and it is a pyroclastic material with mean diameter ranging from 2 to $64 \mathrm{~mm}$.

At the present time, a series of equipment that combines basic operations of filtration and heat exchange simultaneously is being developed. Among these devices, we have the movingbed heat exchanger-filter. ${ }^{4}$

On one hand, packed beds are commonly used as an energy storage system or heat regenerator. For many systems, a packed bed provides an effective means of energy storage. The dynamic response of a sensible energy storage system has been widely studied in the past, and precise predictive models have been developed..$^{5}$ A summary of sensible energy storage models for packed beds have been proposed in refs $6-8$. However, the simultaneous study of the process of gas filtration and heat exchange is very rare. A heat exchanger where solid particulates

* To whom correspondence should be addressed. Tel.: +34 91624 8462. Fax: +3491624 9430. E-mail: dsantana@ing.uc3m.es.

† Universidad de Las Palmas de Gran Canaria.

$¥$ Universidad Carlos III de Madrid. are filtered and a granular filter that recovers energy simultaneously is not mentioned in the Literature Cited section. ${ }^{9}$ This is due to the fact that both polluting hot gas filters and solidgas regenerators have generally been studied, designed, and developed separately.

The purpose of the current work is to conduct a systematic experimental study of aerosol filtration and heat exchange in a fixed-bed heat exchanger-filter (FHEF) using Lapilli as a granular material and to examine the effects of various operating variables such as height of the bed, dust particle concentration, gas temperature, specific deposit, pressure drop, total collection efficiency, and thermal efficiency of the FHEF.

In this paper, a numerical simulation of the processes is shown. We have used the finite element method in the mathematical modeling of the interaction between the processes of gas filtration-heat exchange, which is gaining a greater importance. Thus, we have tried to discover the relationships between experiment and numerical simulations.

Starting from the filtration process, we have simulated variables such as porosity of the filter, specific surface area, and specific deposit of the filter. These variables vary with time because the granular filtration is inherently a nonsteady-state process. Later, we have introduced the results obtained in the equations that represent the heat transfer process. In these equations, we also find porosity variables and specific surface area. In this way, we not only have the solution of the problem but also a complete view of the simulation of the processes of heat exchange and filtration.

\section{Experimental Work}

The equipment used in this experimental work is shown in Figure 1 and consists of the following parts.

Dust-Generating System. A fluidized bed was used as a dust generator to obtain the aerosol used in the experiments. The fluidized bed of $94 \mathrm{~mm}$ i.d. and $178 \mathrm{~mm}$ height was fitted with a perforated-plate distributor. The material of the distributor was stainless steel with a thickness of $0.7 \mathrm{~mm}$. The distributor has been designed using the orifice theory, and the orifice pressure drop is only a small fraction of the total pressure drop. In our 


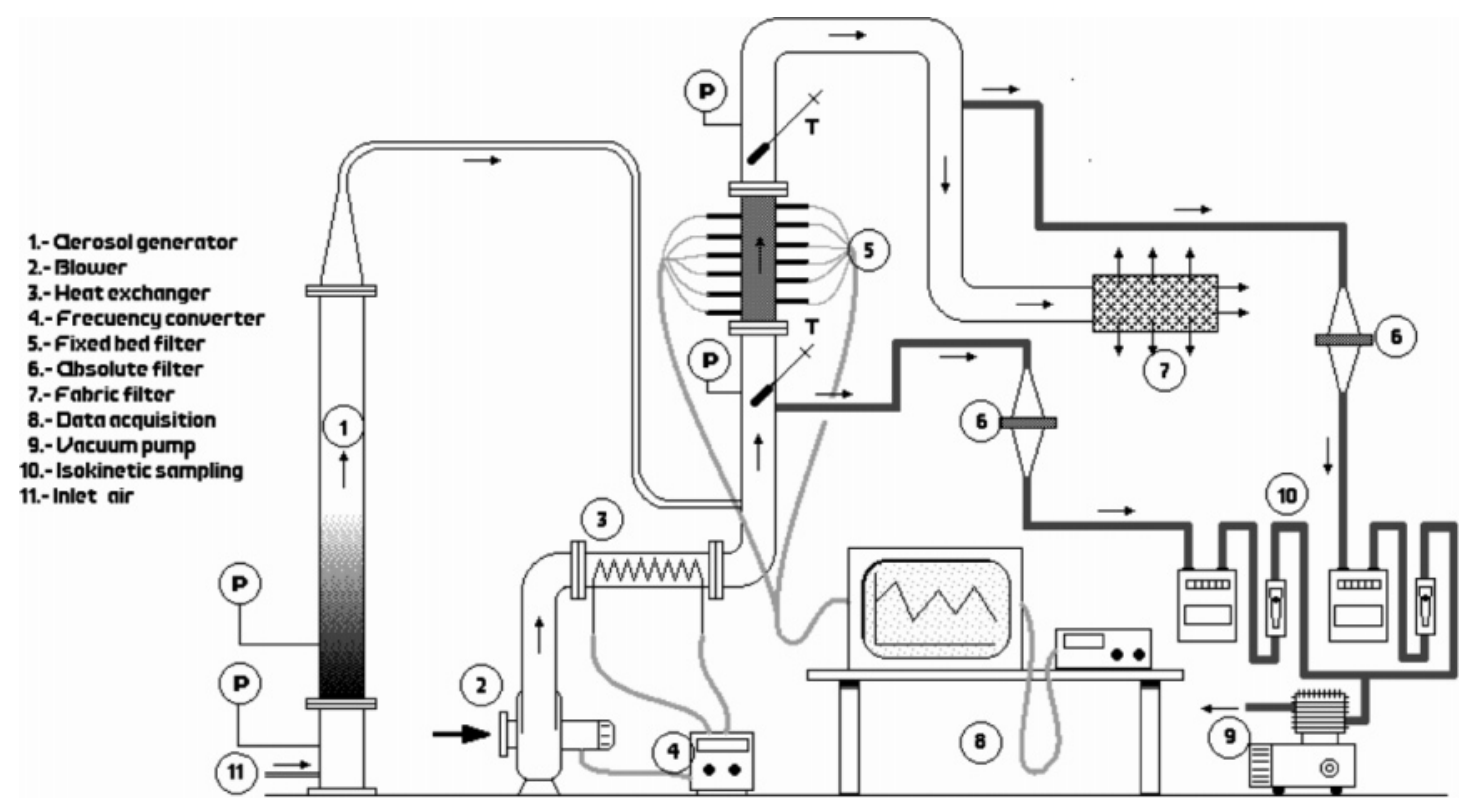

Figure 1. Diagram of the experimental apparatus.

case, we have used the procedure developed by Kunii and Levenspiel, ${ }^{10}$ and we get orifices of $2 \mathrm{~mm}$ of diameter, with an orifice distribution of one orifice for each $3.65 \mathrm{~cm}^{2}$ of distributor surface.

The top of the bed was tapered so that an abrupt increase in gas velocity was avoided. This part of the column was connected with the inlet of the FHEF by means of an antistatic tube to prevent static deposition of the elutriated dust before the process of filtration.

The material used in the fluidized bed was a mixture of coarse sand whose size ranged from 300 to $700 \mu \mathrm{m}$ and fine elutriable particles. The fluidized-bed regime was dominated by the concentration of coarse particles. Then, the fluidized bed operated in the bubbling-free regime in all the experiments. In each test, only one type of fine particles was used, and the fluidization velocity was well-below the terminal velocity of coarse particles, avoiding coarse sand particles elutriation. The fine particles were calcium carbonate with a particle mean size of $4.82 \mu \mathrm{m}$, alumina with a particle mean size of $4.43 \mu \mathrm{m}$, and dust from a central power station with a particle mean size of $15.79 \mu \mathrm{m}$.

The elutriated aerosol escapes from the upper side of the fluidized bed through the poly(vinyl chloride) (PVC) cone connected to the main pipes of the filtration setup by means of an antistatic tube. In this way, the aerosol generated is first added to the main flow of air heated by means of a heat exchanger, according to Figure 1. Then an impulsion takes place from the blower to the FHEF.

It is important to mention that both the aerosol and flow concentrations are controlled by means of a frequency converter that is connected to the blower. Finally, it is important to take into account that the dust generation has been designed according to the experiments provided by Kunii and Levenspiel $^{10}$ and Santana and co-workers. ${ }^{11}$

Fixed-Bed Heat Exchanger-Filter (FHEF). The fixed-bed heat exchanger-filter (FHEF) has been built with a steel tube of $110 \mathrm{~mm}$ i.d., $1 \mathrm{~mm}$ thickness, and $500 \mathrm{~mm}$ length. In the zone destined to the processes of filtration and heat exchange, we fill the granular medium Lapilli, which is covered by a stainless steel net of $0.5 \mathrm{~mm}$ aperture (opening between adjacent wires). This net prevents the Lapilli from going toward other parts of the experimental equipment. The lower net has been fitted by a bridle, and the upper one is adjusted according to the height of the FHEF used in each test. The filter was covered with a tubular fiberglass isolator (Isober type IS-KA-00254601-240) of $43 \mathrm{~mm}$ thickness and covered externally by a sheet of aluminum.

Transient temperature measurements have been taken using chrome-constantan thermocouples. Fifteen thermocouples were placed at different radial positions but at the same axial position, namely, $1 / 5,2 / 5,3 / 5$, and $4 / 5$, from the axis of the bed. At the same time, two thermocouples were placed at different axial positions at the inlet and outlet of the bed. The details of the laying of thermocouples can be seen in Figure 2.

For a FHEF of $15 \mathrm{~cm}$ height, there are four levels of thermocouples, which are equidistant in axial length. In each level, there are three thermocouples out of phase $120^{\circ}$. These ones are placed in the axis of the cylinder (FHEF), in the wall, and in $R / 2$. The different temperatures of the aerosol at the inlet of the FHEF were obtained with a heat exchanger gas - gas by combining sufficiently two electrical resistances of $2200 \mathrm{~W}$ and another of $1100 \mathrm{~W}$ controlled by an Eliwell electronic thermostat (EWDR 905 programmable model) that is equipped with a K-type thermocouple and connected to an electric panel. An infrared thermometer is used to measure the temperature of the Lapilli close to the wall of the FHEF.

The bed consists of particles of Lapilli whose size ranged from 20 to $40 \mathrm{~mm}$, and the bed height $(5-20 \mathrm{~cm}$ ) was varied in terms of the experiment that was carried out. The porosity of the FHEF varied between 0.44 and 0.465 , and the superficial velocity ranged from 0.88 to $1.30 \mathrm{~m} / \mathrm{s}$. Once the granular medium was placed in the inner part of the steel column, it was surrounded by an insulating material. At the outlet of the fixed-bed heat exchanger-filter (FHEF), a fabric filter acted as an absolute filter collecting the dust particles that were not held by the FHEF.

The influent and effluent dust concentrations were determined by isokinetic sampling according to ANSI/ASME norm. ${ }^{12}$ The influent and effluent concentrations were determined by weighing particles collected by a Whatman filter paper No. 1 . Details of the isokinetic sampling are given in Figure 1 and by Henríquez and Macías-Machín. ${ }^{9}$

\section{Model Equations}

The mathematical model, which represents both processes of heat exchange and filtration, was solved by the method of 


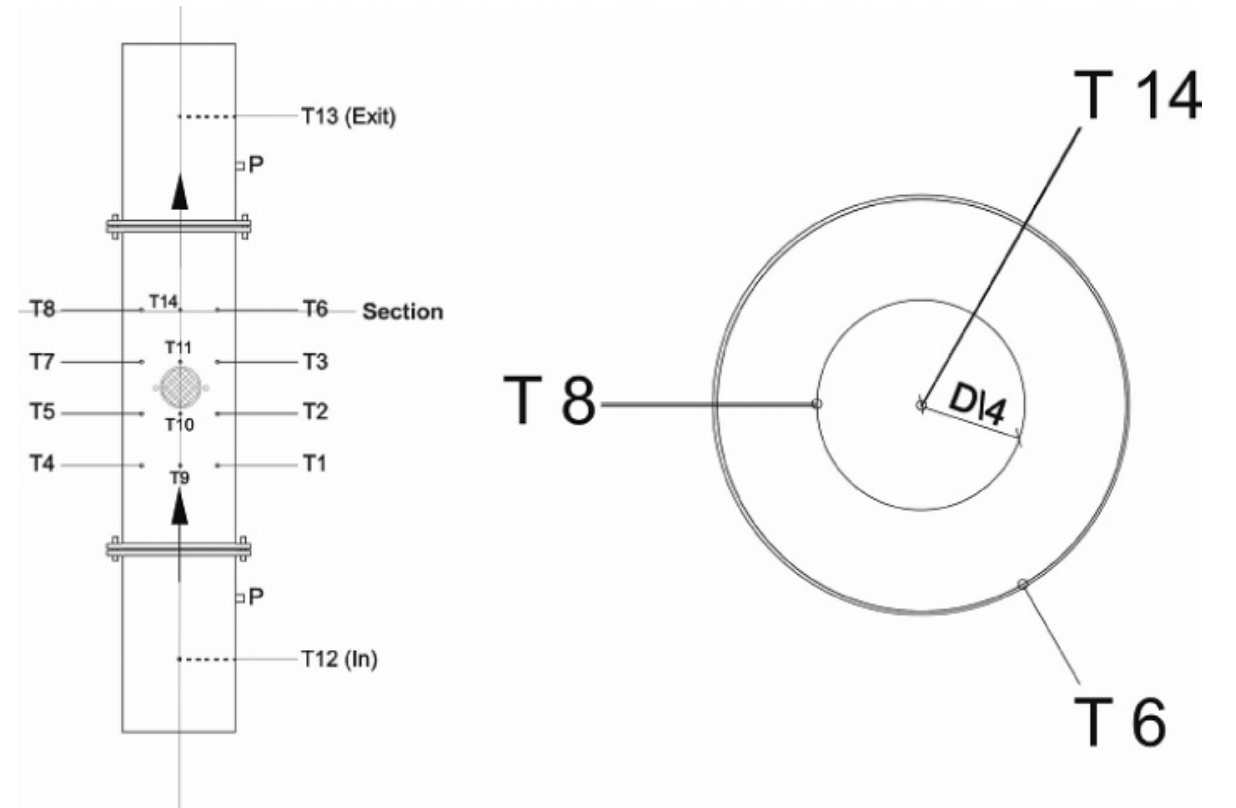

Figure 2. Sectional view of the installation of the thermocouples.

finite elements. In this way, we get the transient response of the FHEF, the variation of specific deposit through the FHEF, and the evolution of the porosity of the bed with time, as well as the variation of the pressure drop in the fixed-bed heat exchanger-filter.

The joint model of filtration-heat exchange is based on the work of Khan and Beasley ${ }^{13}$ as well as those Mizukami and co-workers ${ }^{14}$ and Henríquez and Macías-Machín ${ }^{9}$ for the case of moving beds, taking into account that there is not heat loss around the FHEF.

The equations, which represent the joint process of filtrationheat exchange, are the following:

Heat Transfer. For the fluid,

$$
\begin{aligned}
& \rho_{\mathrm{f}} c_{\mathrm{f}} \frac{\partial T}{\partial t}+\rho_{\mathrm{f}} c_{\mathrm{f}} \frac{u_{0}}{\epsilon} \frac{\partial T}{\partial y}= \\
& k_{\mathrm{eff}}^{y} \frac{\partial^{2} T}{\partial y^{2}}+k_{\mathrm{eff}}^{r}\left(\frac{\partial^{2} T}{\partial r^{2}}+\frac{1}{r} \frac{\partial T}{\partial r}\right)+\frac{h}{\epsilon} S(\theta-T)
\end{aligned}
$$

For the solid,

$$
\rho_{\mathrm{s}} c_{\mathrm{s}} \frac{\partial \theta}{\partial t}=\frac{h}{(1-\epsilon)} S(T-\theta)
$$

The initial and boundary conditions of the problem are as follows:

$$
\begin{array}{ccc}
t=0 & \multicolumn{1}{c}{T=\theta=\theta_{0}} \\
t>0 & y=0 & T=T_{0} \\
r=0 & \forall y \quad \frac{\partial \theta}{\partial r}=0 \\
r=0 & \forall y \quad \frac{\partial T}{\partial r}=0 \\
r=R \quad \forall y & -k_{\mathrm{ew}} \frac{\partial T}{\partial r}=h_{\mathrm{w}}\left(T-T_{\mathrm{w}}\right)
\end{array}
$$

In this model, axial and radial conductivities have been considered and suggested by Wakao and Kaguei. ${ }^{15}$ It is given by the following expression,

$$
k_{\mathrm{eff}}^{y}=k_{\mathrm{eff}}^{0}+\delta P r \cdot R e \cdot k_{\mathrm{f}}
$$

where the fluid phase effective thermal conductivity of the bed containing a stagnant gas was obtained by Kunii and Levenspiel ${ }^{10}$ and is given by the following expression:

$$
k_{\mathrm{eff}}^{0}=\epsilon k_{\mathrm{f}}+(1-\epsilon) k_{\mathrm{s}} \frac{1}{\left(\phi_{\mathrm{B}} \frac{k_{\mathrm{s}}}{k_{\mathrm{f}}}+\frac{2}{3}\right)}
$$

In the same manner, this conductivity in the proximity of the wall is expressed as

$$
k_{\mathrm{eff}, \mathrm{w}}^{0}=\epsilon_{\mathrm{w}} k_{\mathrm{f}}+\left(1-\epsilon_{\mathrm{w}}\right) k_{\mathrm{s}} \frac{1}{\left(\phi_{\mathrm{w}} \frac{k_{\mathrm{s}}}{k_{\mathrm{f}}}+\frac{2}{3}\right)}
$$

In these equations, the equivalent thicknesses of stagnant gas film around contact points between particles, which aids in the transport of heat from particle to particle, and between adjacent particles and the wall surface were obtained by Kunii and Levenspiel. ${ }^{16}$

Then, the heat transfer coefficient at the wall region containing a stagnant gas is given as follows ${ }^{16}$

$$
h_{\mathrm{w}}^{0}=\frac{2 k_{\mathrm{eff}, \mathrm{w}}^{0}}{d_{\mathrm{m}}}
$$

and for a gas flowing through a granular medium, the heat transfer coefficient at the wall is given by

$$
h_{\mathrm{w}}=h_{\mathrm{w}}^{0}+\alpha_{\mathrm{w}} c_{\mathrm{f}} \rho_{\mathrm{f}} u
$$

where $\alpha_{w}$ is a constant that represents the movement and mix of the gas in the wall region ${ }^{17}$ and a reasonable value is 0.05 .

Filtration. A perfect modeling of solid-gas contacting is further complicated because the accumulation of deposited particles during the filtration process causes a continuous change in the structure of the medium. This situation is more complicated in the case of polydispersed aerosol, since the effect of 
deposition is dependent not only on the total amount of deposition but also on the size distribution of the deposited particles. $^{18}$

Difficulty in predicting these parameters and measuring the local void fraction leads one to adopt a formulation of the macroscopic equations for modeling particle collection in the FHEF. An approach involving the macroscopic conservation equations and rate equations that describe the dynamic behavior of granular filtration ${ }^{14}$ can be written as

$$
\begin{gathered}
u \frac{\partial C}{\partial y}+\frac{\partial \sigma}{\partial t}=0 \\
\frac{\partial \sigma}{\partial t}=u \lambda C
\end{gathered}
$$

We have considered a constricted tube model, where the filter coefficient is obtained as

$$
\lambda=\left[\frac{6(1-\epsilon)}{\pi}\right]^{1 / 3} \frac{\eta}{d_{\mathrm{m}}}
$$

and where the porosity variation of the medium is related to $\sigma$ by the following equation

$$
\epsilon=\epsilon_{0}-\left(\sigma / \rho_{\mathrm{d}}\right)
$$

With respect to the unit, collection efficiency can be determined as follows, according to Tien: ${ }^{19}$

$$
\eta=1-\left(C / C_{0}\right)^{1 / N}
$$

On the other hand, the pressure gradient of the gas is given by

$$
-\frac{\mathrm{d} p}{\mathrm{~d} y}=\frac{150}{36} \mu S^{2} \frac{(1-\epsilon)^{2} u}{\epsilon^{3}}+\frac{1.75}{6} \rho_{\mathrm{f}} S \frac{1-\epsilon}{\epsilon^{3}} u^{2}
$$

where the specific surface area of the medium is related to the dustless medium specific surface area and to the dust specific surface area by eq $15^{19}$

$$
S=\frac{1}{1-\epsilon}\left[\left(1-\epsilon_{0}\right) S_{\mathrm{m}}+\frac{\sigma S_{\mathrm{d}}}{\rho_{\mathrm{d}}}\right]
$$

and where the interstitial velocity of the gas can be obtained as

$$
u=\frac{u_{0}}{\epsilon}
$$

Finally, the system of partial differential equations (eqs 9 and 10) was solved with the following initial conditions:

$$
\begin{array}{rl}
y=0 & C=C_{0} \\
t=0 & \sigma=0
\end{array}
$$

The solution of eqs 9 and 10 with the initial and boundary conditions gives the effluent history as well the specific deposit in the bed. To obtain the solution of eqs 9 and 10, the filter coefficient $\lambda$ must be known, and it is necessary for the interpretation of experimental data.

\section{Numerical Solution}

The joint process of heat transfer and filtration is solved by means of the finite element method. To solve the equation of the process fluid, its equation in implicit form becomes as follows:

$$
\begin{array}{r}
\rho_{\mathrm{f}} c_{\mathrm{f}} \frac{\partial T}{\partial t}+\rho_{\mathrm{f}} c_{\mathrm{f}} \frac{u_{0}}{\epsilon} \frac{\partial T}{\partial y}-k_{\mathrm{eff}}^{y} \frac{\partial^{2} T}{\partial y^{2}}-k_{\mathrm{eff}}^{r}\left(\frac{\partial^{2} T}{\partial r^{2}}+\frac{1}{r} \frac{\partial T}{\partial r}\right)- \\
\frac{h}{\epsilon} S(\theta-T)=0
\end{array}
$$

The transformations that are made in this equation, to come to the calculation of the fluid temperatures by the last method, lead to the variational formulation of the problem, with the following structure:

$$
\begin{gathered}
\int_{\Omega} \rho_{\mathrm{f}} c_{\mathrm{f}} \frac{\partial T}{\partial t} N_{\mathrm{T}} \mathrm{d} \Omega+\int_{\Omega} \rho_{\mathrm{f}} c_{\mathrm{f}} \frac{\vec{u}_{0}}{\epsilon} \nabla T N_{\mathrm{T}} \mathrm{d} \Omega- \\
\int_{\Omega} k_{\mathrm{eff}}^{y} \nabla T \nabla N_{\mathrm{T}} \mathrm{d} \Omega-\int_{\Omega} k_{\mathrm{eff}}^{r} \nabla T \nabla N_{\mathrm{T}} \mathrm{d} \Omega- \\
\int_{\Omega} k_{\mathrm{eff}}^{r} \frac{1}{r} \nabla T N_{\mathrm{T}} \mathrm{d} \Omega+\int_{\Omega} h \frac{S}{\epsilon}(T-\theta) N_{\mathrm{T}} \mathrm{d} \Omega=0
\end{gathered}
$$

The temporal term $\rho_{\mathrm{f}} c_{\mathrm{f}}(\partial T / \partial t)$ is determined with the indicated structure by the first integral to the domain $\Omega$. In our case, the FHEF is cylindrical, by symmetry, and can make a simplification in the problem, transforming the domain to rectangular coordinates. ${ }^{20}$ Where $N_{\mathrm{T}}$ are the named shape functions for the temperature of gas, that is, where they agree with the interpolation functions used in the program for such a variable, since the method of Galerkin is used, in the weighted functions or form in the method of weighed residuals, for the problem resolution.

The forced convection of the problem, adopts the form formulated in the second integral, extended to the same domain. With respect to the diffusive term in the $y$ direction, Green's theorem ${ }^{21}$ was applied. On the other hand, the term $-k_{\text {eff }}^{r} \partial^{2} T /$ $\partial r^{2}$ represents the radial diffusive behavior of the fluid: it receives the same mathematical treatment as in the previous case, so that it is represented with another integral. In the same manner, $-K_{\text {eff }}^{r}(1 / r)(\partial T / \partial r)$ is transformed into the fifth term of the integral. Finally, the last term in the equation takes into account the heat transfer between solid particles and gas by convection.

Analogous to the last development for the gas phase, the differential equation that governs the solid-phase (Lapilli) temperature is given by

$$
\rho_{\mathrm{s}} c_{\mathrm{s}} \frac{\partial \theta}{\partial t}-\frac{h}{(1-\epsilon)} S(T-\theta)=0
$$

and the transformations lead to the following expression

$$
\int_{\Omega} \rho_{\mathrm{s}} c_{\mathrm{s}} \frac{\partial \theta}{\partial t} N_{\theta} \mathrm{d} \Omega+\int_{\Omega} h \frac{S}{1-\epsilon}(\theta-T) N_{\theta} \mathrm{d} \Omega=0
$$

where $N_{\theta}$ is the shape function associated to the method of Galerkin for the solid phase.

The boundary conditions of the problem are affected by the procedure followed in the problem resolution, in effect, for the fluid; they are contemplated in the integral that is exposed next:

$$
\int_{\partial \Omega} h_{\mathrm{w}} \epsilon\left(T-T_{w}\right) N_{\mathrm{T}} \mathrm{d} l
$$

In this case, it is an integral extended to the domain that surrounds $\Omega$, that we denominated $\partial \Omega$.

In the same manner, for the solid (Lapilli), the boundary can be expressed by the following equation: 


$$
\int_{\partial \Omega} h_{\mathrm{w}}(1-\epsilon)\left(\theta-T_{\mathrm{w}}\right) N_{\theta} \mathrm{d} l
$$

For the case of the filtration process starting off of the differential eqs 9 and 10, and using the finite elements method, this equation is transformed to the implicit form:

$$
\frac{\partial \sigma}{\partial t}-u \lambda C_{0} \mathrm{e}^{-\lambda y}=0
$$

For its resolution, the weighted residual method is used, associated with Galerkin's method to incorporate the necessary shape functions for the numerical resolution of the problem by finite elements. Thus, the variational formulation of the problem is reflected with the following expression:

$$
\int_{\Omega} \frac{\partial \sigma}{\partial t} N_{\sigma} \mathrm{d} \Omega-\int_{\Omega} \lambda C_{0} u_{0} \mathrm{e}^{-\lambda y} N_{\sigma} \mathrm{d} \Omega=0
$$

Once the equations for the filtration process are solved, the variables are replaced in the representative equations of the heat transfer process (porosity, specific surface, etc.), which allows for the solution of the joint process of filtration-heat transfer.

For the numerical resolution of the joint process of filtration and heat transfer, mesh in the domain $\Omega$ of the FHEF with triangular finite elements with a characteristic length of $3 \mathrm{~mm}$ gave rise to 1022 nodes and 2041 triangles. In the nodes of each triangular element, the program must calculate three unknown variables: the fluid temperature, the solid temperature, and the specific deposit. A $0.5 \mathrm{~s}$ time step was chosen, compatible with the problem resolution, since the set of implicit differential equations that define the processes of filtration and heat transfer are unconditionally stable. ${ }^{22}$ To solve the problem, the method of the generalized residuals, denominated briefly G.M.R.E.S., was chosen. For its resolution, the numerical library elaborated by Dular and Geuzaine ${ }^{22}$ has been used.

In the resolution of the differential equations in the time domain, of the joint process of filtration-heat transfer, the directives marked in ref $23-25$ were taken into consideration. For the resolution of the problem, we have chosen the complete implicit method, since it is adapted to the structure of our work. In the problem domain, the resources for mesh developed by Geuzaine and Remacle ${ }^{25}$ have been used.

\section{Results and Discussion}

The performance of a filter can be described by its overall collection efficiency. To obtain such a variable, it is necessary to measure the influent and effluent particle fine concentrations. Normally, we can express the dynamic behavior of the filters by the evolution of $E$ with time or with respect to the mass of particles collected per bed volume or specific deposit. The latter quantity can be evaluated by eq 28 . The overall collection efficiency, however, does not provide a direct measurement of the particle-collecting capability of the filter bed. ${ }^{9}$

The quantity found to be useful in characterizing the particlecollecting capability of the FHEF is the so-called filter coefficient, and this variable is related to the unit collection efficiency. It is important to note that $\eta$ can be obtained from experimental data. With the values of the influent and effluent concentrations, $\eta$ can de determined. The relationship between these variables provides a meaningful indicator of the intrinsic ability of grain to capture particles. The total collection efficiency of the filter at any time is defined as

$$
E=\frac{C_{0}-C}{C_{0}}
$$

Generally, we can express the dynamic behavior of the filters by the variation of $E$ with time or by the specific deposit. Experimentally, $\sigma$ can be obtained as ${ }^{19}$

$$
\sigma=m /(A H)
$$

Analogously, the specific deposit can be evaluated as

$$
\sigma=\frac{\int_{0}^{t}\left(C_{0}-C\right) u A \mathrm{~d} t}{A H}=\frac{u}{H} \int_{0}^{t}\left(C_{0}-C\right) \mathrm{d} t
$$

For the process of heat transfer in the FHEF, it is important to know the thermal efficiency, $E_{\mathrm{t}}$, is defined as

$$
\begin{aligned}
& E_{t}=(\text { heat taken by cold solids in time } t / \\
& \text { maximum possible take-up in time } t)= \\
& \text { (heat lost by hot gas in time } t / \\
& \text { maximum possible heat lost in time } t \text { ) }
\end{aligned}
$$$$
E_{t}=\left(\Delta T_{\mathrm{h}} / \Delta T_{\max }\right)=\left[\left(T_{\mathrm{h}, \text { in }}-T_{\mathrm{h}, \text { out }}\right) /\left(T_{\mathrm{h}, \text { in }}-T_{\mathrm{s}}\right)\right]_{\mathrm{gas}}
$$

We assume that heats losses to surroundings are negligible, and that the mean physical properties are independent of the temperature. Also, assume constant mass flow rate and gas inlet temperature.

Influence of the Height of the FHEF. The results shown in Figure 3 were obtained at three different heights of the FHEF $(5,10$, and $15 \mathrm{~cm})$ and a fixed gas velocity of $1.11 \mathrm{~m} / \mathrm{s}$. These experiments were made at ambient temperatures, and the filtration efficiencies varied between 78 and $96 \%$. We used a granular medium (Lapilli) of $2 \mathrm{~mm}$ in the filtration process, and the mean diameter of the dust $\left(\mathrm{CaCO}_{3}\right)$ was $4.82 \mu \mathrm{m}$. The data plotted for this gas velocity showed that the filtration efficiency increases when the height of the FHEF increases.

It has been noted that there is a remarkable difference between the efficiencies of the beds at different heights due to both clogging effects and the increase of the specific deposit with the height $(H=15 \mathrm{~cm})$ of the fixed-bed heat exchanger-filter (FHEF). These findings coincide with the studies done by Pendse and Tien. ${ }^{26}$

In Figure 3, it can be observed that, for three different heights of the FHEF, the dust retention on the packing (Lapilli) of the granular filter increases with time. This is due to the fact that the particles of $\mathrm{CaCO}_{3}$ settle easily at the end of the filtration process.

In Figure 4, we can observe the pressure drop with regard to time for different filter heights of the granular bed. These experiments were obtained at room temperature $\left(20^{\circ} \mathrm{C}\right)$, and the bed heights were 5,10 , and $15 \mathrm{~cm}$. It can be observed that pressure drop for each height of the FHEF increases in a linear way with the operation time. This fact agrees with Ergun's equation $^{27}$ that was developed for pressure drop of all kinds of packed beds.

Influence of Dust Sizes. The influence of the dust sizes on the overall collection efficiency, $E$, of the FHEF has been plotted as a function of time in Figure 5. We have used dust from a thermal power station with a mean diameter of $15.79 \mu \mathrm{m}$. Also, we have used $\mathrm{Al}_{2} \mathrm{O}_{3}$ with a particle mean diameter of $4.43 \mu \mathrm{m}$.

In Figure 5, it is observed that the overall collection efficiency increases when the size of the dust increases, verifying that the grade of efficiency decreases when particle size decreases. 


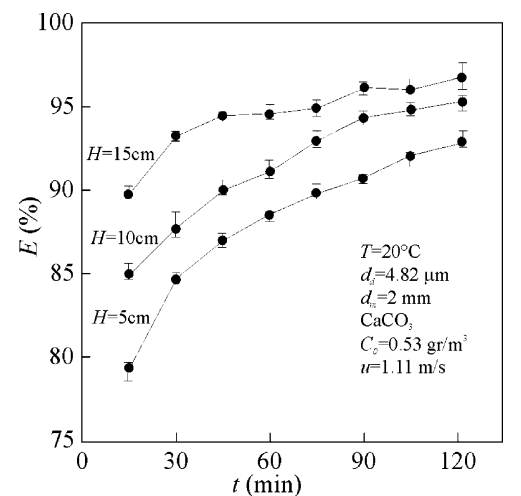

Figure 3. Efficiency of the FHEF at three bed heights.

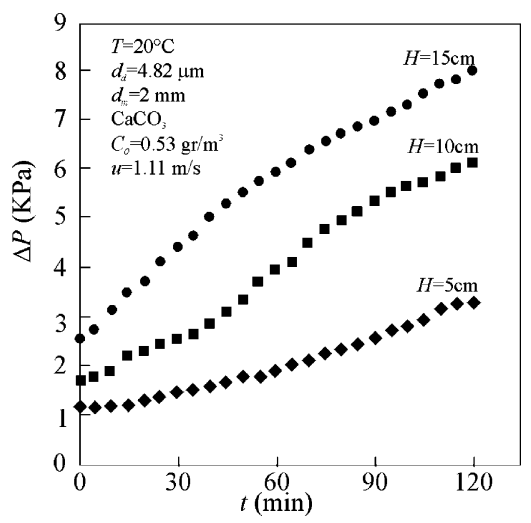

Figure 4. Pressure drop of the FHEF.

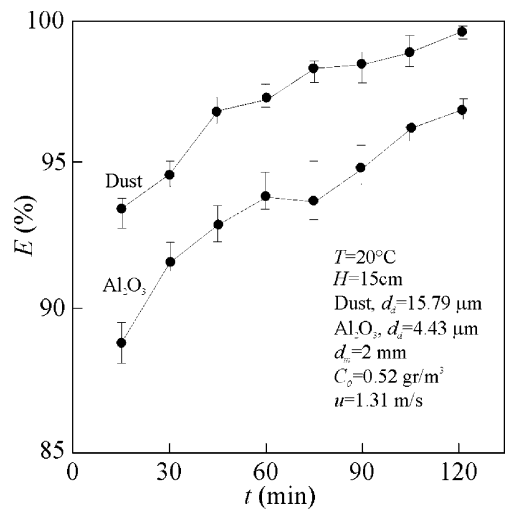

Figure 5. Efficiency of the FHEF at two aerosol sizes.

Because of this, as the particles size becomes smaller, they will pass more easily through the fixed-bed heat exchanger-filter (FHEF), and it is observed that the overall collection efficiency for the $\mathrm{Al}_{2} \mathrm{O}_{3}$, with a mean diameter of $4.43 \mu \mathrm{m}$, is smaller than that for the dust (central power station).

Influence of the Dust Concentration. To study the effect of the dust concentration on the filtration process in the FHEF, the following dust concentrations were generated: $0.12,0.54$, and $1 \mathrm{~g} / \mathrm{m}^{3}$. These concentrations were later filtrated in the FHEF.

In Figure 6, it is observed that the global efficiency of filtration, $E$, increases when the dust concentration also increases. This result is logical, because an increase in the dust concentration gives place to a progressive increase in the specific deposit. Besides, the porosity of the granular filter decreases in time. Then, the formation of a cake takes place as well as the clogging of the granular filter. ${ }^{28,29}$ The appearance of these two processes depends on the dust concentration used in the filtration process of the polluting gases. It can be observed that the higher

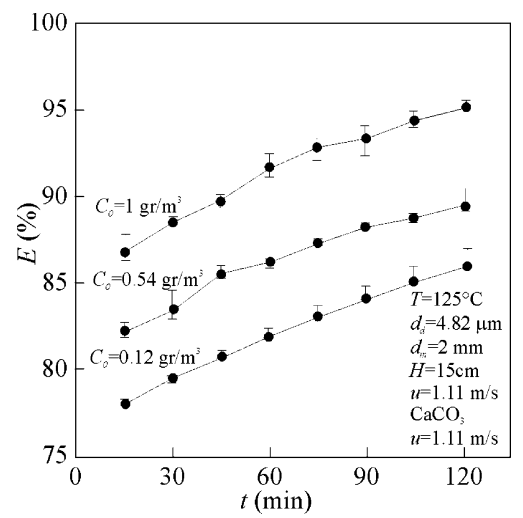

Figure 6. Efficiency of the FHEF at three dust concentrations.

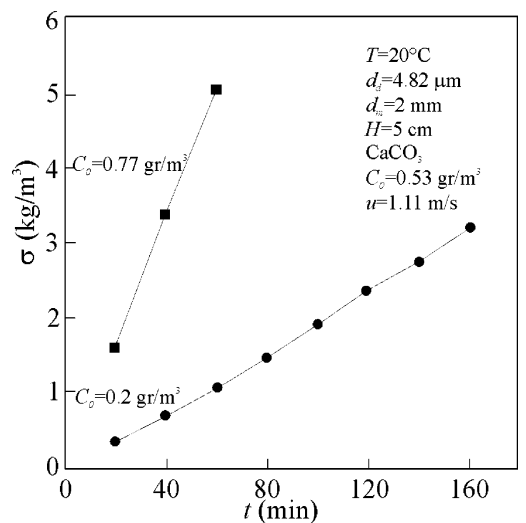

Figure 7. Specific deposit $\sigma$ for two concentrations of dust.

the value of concentration, the sooner the phenomenon of clogging appears. ${ }^{30}$

The effect of dust concentration on the process of particle collection is a very important issue. In Figure 7, the specific deposit is given as a function of time. The data shown in this figure was obtained for the same height of the bed $(H=5 \mathrm{~cm})$, and we have observed that, if the dust concentration decreases $\left(C_{0}=0.2 \mathrm{~g} / \mathrm{m}^{3}\right)$, we can obtain a higher operation time of the FHEF because the clogging effect decreases with time.

The results obtained from the extent deposition of the dust described by the specific deposit (Figure 7) can be used to study the dynamic behavior of the granular filter of Lapilli. A study to predict the dynamic behavior of the deep bed filters is developed by Chiang and Tien ${ }^{31}$ where they demonstrated the significant effect of the specific deposit on the filter performance.

Influence of the Gas Temperature on the Filtration Process. The joint process of filtration-exchange heat can be observed in Figure 8 for a polluting gas velocity at the inlet of $1.11 \mathrm{~m} / \mathrm{s}$. The same concentration has been used for all the experiments $\left(C_{0}=0.536 \mathrm{~g} / \mathrm{m}^{3}\right)$. The temperatures of gas + dust have been as follows: $20,95,125$, and $180{ }^{\circ} \mathrm{C}$.

In all the tests that were performed, we have observed that the global efficiency of filtration for a given temperature increases with time until it reaches a maximum. Later it almost stabilizes. This is due to the fact that the FHEF is free of particles at the beginning of the process and the void fraction of the bed is higher.

As operation time goes on, a cake is formed at the inlet of the granular filter, causing an increase not only in particle accumulation but also in specific deposit of FHEF. Detailed studies on the cake formation based on particle addition as well as its modeling are given by Stamakis and Tien. ${ }^{32}$ 


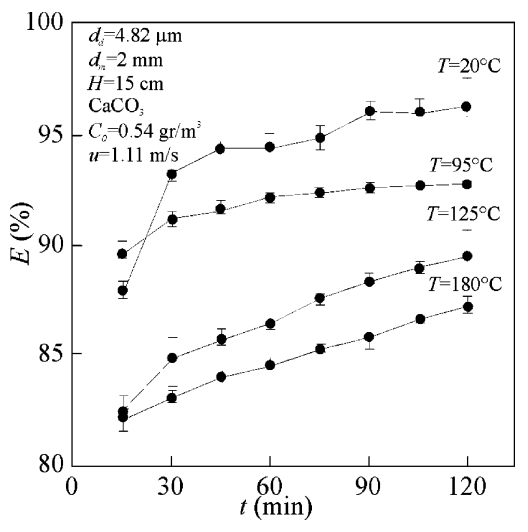

Figure 8. Total collection efficiency at different temperatures.

The influence of the different temperatures on overall collection efficiency, $E$, of the FHEF has been plotted as a function of time in Figure 8. We have used a dust of $\mathrm{CaCO}_{3}$ with a particle mean diameter of $4.82 \mu \mathrm{m}$. It is observed that the grade of efficiency decreases with increasing temperature, in agreement with filtration theory, since the viscosity of the gas increases with temperature and because the Cunningham factor slip correction for particles $>1 \mu \mathrm{m}$ can be approximated to $C u=1 .{ }^{33}$ Also, the collection efficiency decreases when the temperature increases due to the decreasing value of the inertial parameter (Stokes number). On the other hand, for particles $>$ $1 \mu \mathrm{m}$, in the absence of an applied external force, one of the major mechanisms for aerosol collection in granular filtration is inertial impaction. ${ }^{19}$

In Figure 8, we have the overall efficiency of filtration of the FHEF for different temperatures, and we have observed with respect to the filtration curves that the maximum efficiency is acquired at room temperature, even when the concentration of dust was the same for all the experiments.

Modeling. The gas - solid contact established inside a FHEF is very complex because the granular medium (Lapilli) has interstitial voids. Dusty gas flows through the filter, the particles collect on the granular medium, where it has interstitial voids with several dynamic shapes. A perfect modeling of solid-gas contacting is further complicated because the accumulation of deposited particles during the filtration process causes a continuous change in the structure of the medium.

Difficulty in predicting the parameters of the modeling, for example, the local void fraction, leads one to adopt a formulation of the macroscopic equations for modeling particle collection in the FHEF.

On the other hand, with respect to the process of heat transfer, it is necessary to determine the two-dimensional effects on the FHEF response; an accurate model of the transient temperature variations in the axial and radial directions during the process of filtration-heat exchange is required. The two-dimensional computational model of the FHEF used in this study is an extension of an existing filtration-heat exchange model developed by Henríquez and Macías-Machín. ${ }^{9}$ The model employs appropriately governing equations for the fluid and solid phases in the case of heat transfer and macroscopic equations for modeling particle collection that describe the dynamic behavior of granular filtration. ${ }^{19}$

Therefore, if the particle concentration is expressed on a volume basis, the filtration rate is then given as the volume of particle collected per unit filter volume per unit time. Similarly, one may describe the extent of the deposition by introducing the specific deposit expressed as the volume of particle deposited

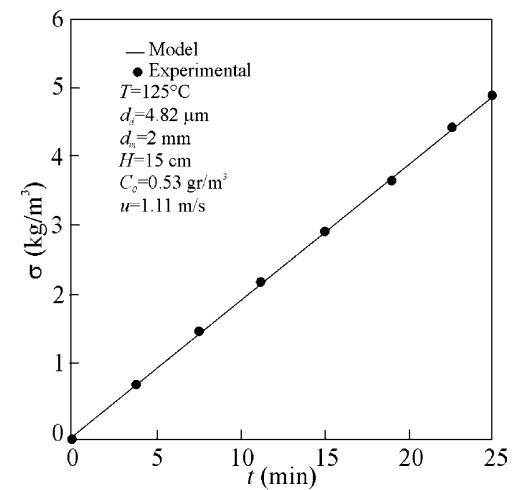

Figure 9. Experimental data and modeling of the specific deposit $\sigma$.

per unit filter volume where the specific deposit is composed of all particles types deposit in the FHEF.

On the basis of experimentally determined values of the effluent and influent concentrations and using the uniform deposition assumption, one can obtain the value of $\sigma$, which can be calculated from eqs 27 and 28 .

The values of the specific deposit $\sigma$ obtained experimentally and those estimated from the modeling were compared with the results also shown in Figure 9. The figure illustrates the predicted value of the specific deposit with the experimental measurements from different operating conditions. The constricted tube model is used to characterize the filter medium, ${ }^{19}$ because the constricted tube model describes more realistically the process of filter clogging. For this reason, one may argue that the constricted tube model, among all the proposed models in the Literature Cited section (isolated sphere model, Kuwabara's model, or Happel's model), is potentially more suitable for studying the transient behavior of granular filtration. Figure 9 demonstrates favorable agreement between the experimental data and the model predictions.

In the past few years, a number of mathematical models have been proposed for estimating the porosity of a particle mixture. ${ }^{34-36}$ In general, these models are only applicable to spherical particles packed under either loose or dense random packing conditions where the particle size and initial porosity is clearly defined. ${ }^{37}$ However, the particles involved in the FHEF are usually not spherical, and particle shape has a strong effect on porosity.

Therefore, in our case, we have considered the effect of deposited particles not in terms of individual deposited particles upon the filter performance but rather in terms of the overall influence. The results of the void fraction using dust $\left(\mathrm{CaCO}_{3}\right)$ with a mean size of $4.82 \mu \mathrm{m}$ as a function of time in a FHEF are shown in Figure 10, and we have used the assumption that the deposition is uniform through the experimental filter. ${ }^{38}$ The results shown in Figure 10 indicate that void fraction of the bed hash favorable agreement with the model predictions.

Finally, the data obtained from the dynamic behavior of granular filtration is required to know the variation of void fraction and specific surface area with time for one entire filtration period. We have determined the model of heat transfer because the variables appear in the simultaneous processes of filtration-heat exchange.

Two-dimensional effects on the dynamic response of the FHEF are the results of the nonuniformity of flow across the filter cross section, combined with the increased average void fraction and also the specific surface area, because the deposition of particles increases with time, also including an increased average void fraction in the near-wall region. To demonstrate these effects, results from previous comparisons between the 


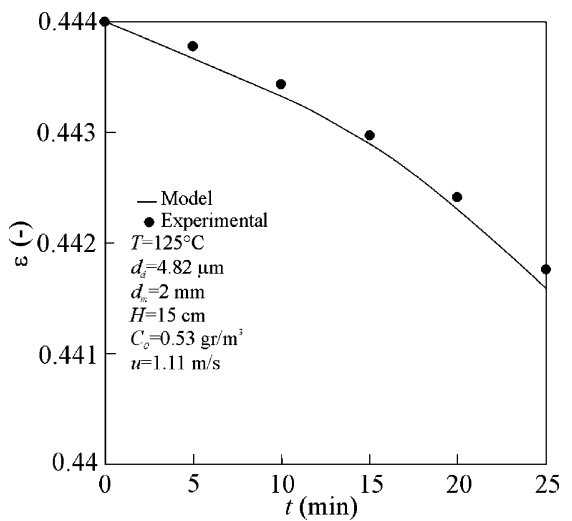

Figure 10. Experimental data and modeling of the void fraction, $\epsilon$.

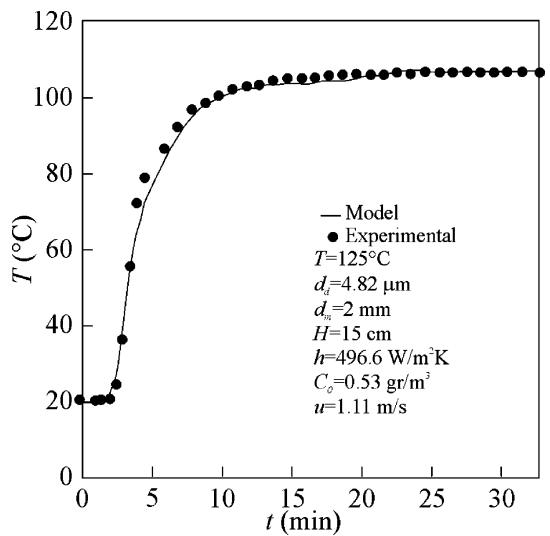

Figure 11. Measured and computed fluid temperature at centerline of the FHEF for a height of $4 / 5$.

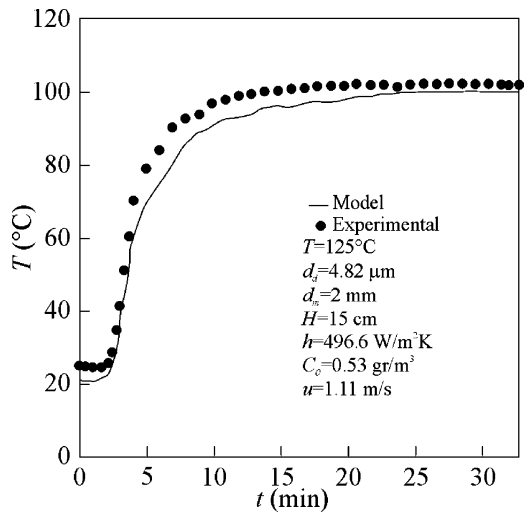

Figure 12. Measured and computed fluid temperature at half of the $R / 2$ of the FHEF of the height of $4 / 5$.

present model and the experimental results will be presented. The results of the 2-D model of filtration and heat exchange are compared with experimental measurements from different operation conditions. The experimental bed provides detailed transient temperature distributions during the processes of filtration-heat exchange.

Figure 11 compares the fluid temperature at the center of the FHEF with the prediction of the model. The results demonstrate favorable agreement between the experimental data and the predicted numerical values. The best agreement obtained for the model is in the centerline of the FHEF; we have an relative error of $2.62 \%$, while in terms of the agreement in the thermocouples situated at half of the radio of the FHEF (Figure 12 ), the error between the experimental data and the model is $4.89 \%$.

Figure 12 shows the fluid temperature of the processes of filtration-heat exchange at half of the radio of the FHEF in

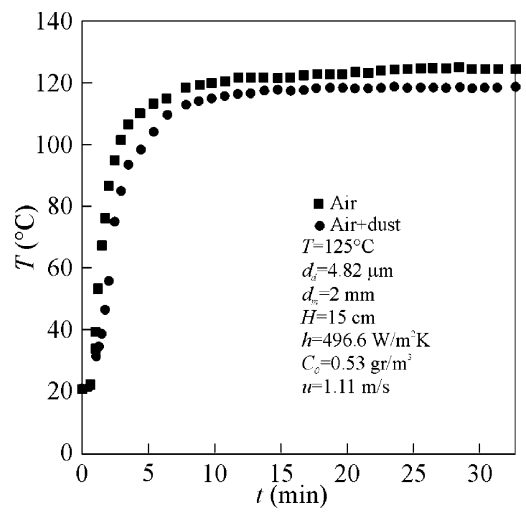

Figure 13. Comparison of the fluid temperatures with dust and without dust at 2/5 of height of the FHEF.

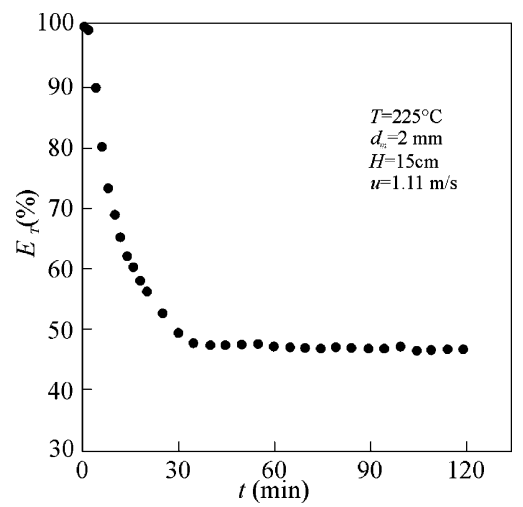

Figure 14. Thermal efficiency of the FHEF.

the present experimental bed, which we compared with the values predicted by the modeling. Numerical experiments with the model indicate that, over a reasonable range of the $k_{\text {eff }}$ values, this property has a negligible effect on the shape of the temperatures curves for the processes of filtration-heat exchange.

Figure 13 indicates a comparison of the fluid temperature at $2 / 5$ of the height of the FHEF, but in this case, we are working with the fluid at the same experimental conditions but with aerosol $\mathrm{CaCO}_{3}$ and without aerosol. The effect of the aerosol creates an entirely different character of the thermal response in the FHEF. Although the influence of changes in the heat transfer coefficient is small, it is clear that the axial thermal dispersion is too great for the nominal $h$ values. However, the form of the present model to predict both temperature distributions in the FHEF incorporates the effect of a new thermal resistance through the granular material (Lapilli); this resistance is the deposition of the dust on the granular material, which influences, notably, the process of heat transfer.

Figure 13 shows the transient response of these two cases, where the dust has a significant effect due to new thermal resistance that has been introduce in the FHEF. The numerical experiments with the model indicate that, over a reasonable range of $k_{\text {eff }}$ values, this resistance is small in the case that we have deposition of the dust in the processes of filtration-heat exchange. It is necessary to find a new equation for $k_{\text {eff }}$, as can be observed in Figure 13, where we have the fluid temperatures with dust and without dust measured and predicted for the numerical model.

Figure 14 shows the thermal efficiency of the FHEF according to Levenspiel ${ }^{39}$ for a gas velocity of $1.11 \mathrm{~m} / \mathrm{s}$. We have observed that, for small sizes of the granular material ( $2 \mathrm{~mm}$ ), a maximum thermal efficiency occurs. When the size of the Lapilli decreases, the thermal efficiency increases, but we have observed that, for 
the size of the Lapilli used ( 2.3 and $4 \mathrm{~mm}$ ), the influences of changes in $E_{\mathrm{T}}$ is very small (3\%). Finally, we can conclude the possibility to use the Lapilli as granular material for storage thermal energy in the FHEF.

\section{Conclusions}

This study identifies the effects of gas velocities, height of the bed, dust concentration, dust particle size, and size of the granular material on the dynamic response of the fixed-bed heat exchanger-filter (FHEF) that uses a granular medium (Lapilli) subjected to an arbitrary time-varying inlet temperature, for both processes of filtration and heat exchange.

Measured values of the overall collection efficiency in different experiments indicate that $E$ decreases when the temperature increases; this question is in agreement with the filtration theory.

We can conclude the possibility to use the Lapilli as a granular material in a fixed-bed heat exchanger-filter (FHEF), because we can improve the overall collection efficiency if we increase the filter height or if we increase the dust particle size of the aerosol used in the experiments.

A predictive numerical model using finite element method was developed that incorporates variations in void fraction, specific surface area, velocity, and transport coefficients due to the combined processes of filtration-heat exchange.

The model is capable of predicting the 2-D transient behavior of the FHEF under various experimental conditions. The model is verified by comparisons with experimental data from the combined processes of filtration-heat exchange.

\section{Acknowledgment}

One of us (D.S.) wishes to acknowledge financial support of the Spanish Ministry of Education under Project No. ENE200601401/ALT.

\section{Nomenclature}

$A=$ cross-sectional area of filter $\left(\mathrm{m}^{2}\right)$

$C=$ effluent dust concentration in the gas $\left(\mathrm{kg} / \mathrm{m}^{3}\right)$

$C_{0}=$ influent dust concentration in the gas $\left(\mathrm{kg} / \mathrm{m}^{3}\right)$

$c=$ specific heat of gas $(\mathrm{J} /(\mathrm{kg} \mathrm{K}))$

$d=$ diameter $(\mathrm{m})$

$E=$ overall collection efficiency

$E_{\mathrm{T}}=$ thermal efficiency

$H=$ height of the filter (m)

$k=$ thermal conductivity of the fluid (W/(m K))

$m=$ mass of particles collected in the filter $(\mathrm{kg})$

$N=$ total number of unit bed elements in series of equation 13

$\operatorname{Pr}=$ Prandtl number $\left(c_{\mathrm{f}} \mu / k_{\mathrm{f}}\right)$

$r=$ radial coordinate $(\mathrm{m})$

$R e=$ Reynolds particle number $\left(d_{\mathrm{m}} u \rho_{\mathrm{f}} / \mu\right)$

$S=$ specific surface $\left(\mathrm{m}^{-1}\right)$

$t=$ time $(\mathrm{s})$

$T=$ gas temperature $(\mathrm{K})$

$u=$ gas velocity $(\mathrm{m} / \mathrm{s})$

$y=$ axial coordinate $(\mathrm{m})$

\section{Greek Symbols}

$\alpha_{\mathrm{w}}=$ constant in equation 8

$\delta=$ Wakao coefficient in equation 4

$\Omega=$ integral domain

$\Delta P=$ pressure drop $(\mathrm{Pa})$

$\phi_{\mathrm{B}}=$ ratio of an equivalent thickness of gas film referring to particle diameter, equation 5 $\phi_{\mathrm{w}}=$ ratio of an equivalent thickness of gas film referring to particle diameter in the vicinity of the wall, equation 6

$\lambda=$ filter coefficient $\left(\mathrm{m}^{-1}\right)$

$\epsilon=$ bed porosity

$\eta=$ unit collector efficiency

$\mu=$ fluid viscosity $(\mathrm{kg} /(\mathrm{m} \mathrm{s}))$

$\rho=$ density $\left(\mathrm{kg} / \mathrm{m}^{3}\right)$

$\sigma=$ specific deposit of the filter $\left(\mathrm{kg} / \mathrm{m}^{3}\right)$

$\theta=$ solid temperature $(\mathrm{K})$

\section{Subscripts}

$0=$ initial or inlet

$\mathrm{d}=$ dust

eff $=$ effective

$\mathrm{f}=$ fluid

$\mathrm{m}=$ dustless medium

$\mathrm{s}=$ solid

$\mathrm{w}=$ wall

Superscripts

$0=$ filter bed containing a stagnant gas

$r=$ radial direction

$y=$ axial direction

\section{Literature Cited}

(1) Tsubaki, J.; Tien, C. Gas filtration in granular moving beds-An experimental study. Can. J. Chem. Eng. 1988, 66, 271.

(2) Socorro, M.; Macias-Machin, A.; Verona, J. M.; Macias, M. Gas filtration using a new granular material. J. Filtr. Soc. 2005, 5, 110.

(3) Gonzalez de Vallejo, L. I.; Jiménez Solas, J. A.; Leguey Jiménez, S. Engineering geology of the tropical volcanic soils of La Laguna, Tenerife. Eng. Geol. 1981, 17, 1.

(4) Jariz, E.; Estevez, A.; Cuellar, J.; Macias-Machin, A. Spanish Patent 9102728, 1991.

(5) Beasley, D. E.; Clark, J. Transient response of a packed bed for thermal energy storage. Int. J. Heat Mass Transfer 1984, 27, 1659.

(6) Gunn, D. J.; Vortmeyer, D. Heat transfer in fixed bed chemical reactors. Proc. 7th Int. Heat Transfer Conf. 1982, B5, 13.

(7) Borlink, J. G. H.; Westertep, K. R. Influence of tube and particle diameter on heat transfer in packed beds. AIChE J. 1992, 38, 703.

(8) Peters, P. E.; Schiffino, R. S.; Harriot, P. Heat transfer in packedtube reactors. Ind. Eng. Chem. Res. 1988, 27, 226.

(9) Henríquez, V.; Macías-Machín, A. Hot gas filtration using a moving bed heat exchanger-filter (MHEF). Chem. Eng. Process. 1997, 36, 353.

(10) Kunii, D.; Levenspiel, O. Fluidization Engineering; ButterworthHeinemann: Stoneham, MA, 1991.

(11) Santana, D.; Rodriguez, J. M.; Macias-Machin, A. Modelling fluidized bed elutriation of fine particles. Powder Tech. 1999, 106, 110

(12) ANSI/ASME Norm; American Society of Mechanical Engineers (Eds.): New York, 1981

(13) Khan, J. A.; Beasley, D. E. Two-dimensional effects response of packed bed regenerators. J. Heat Transfer 1989, 111, 328.

(14) Mizukami, S.; Wakabayashi, M.; Murata, H. Interaction between pressure drop of gas and flow of medium a moving granular filter. Part. Sci. Technol. 1987, 5, 131 .

(15) Wakao, N.; Kaguei, S. Heat and mass transfer in packed beds; Gordon and Breach Science Publishers: New York, 1982.

(16) Kunii, D.; Levenspiel, O. A general correlation for the heat transfer coefficient at wall surfaces of gas-solid contactors. Ind. Eng. Chem. Res. 1991, 30, 136.

(17) Yagi, S.; Kunii, D. Studies of heat transfer near wall surfaces in packed bed. AIChE J. 1960, 6, 97.

(18) Jung, Y.; Tien, C. Granular filtration of polydispersed aerosol. 6th World Filtr. Congr., Nagoya, Japan 1993, 504.

(19) Tien, C. Granular filtration of aerosol and hydrosol; Butterworth: Boston, MA, 1989

(20) Socorro, M. Simulación y experimentación con un filtro-intercambiador granular de lecho fijo. Ph.D. Thesis, Universidad de Las Palmas de Gran Canaria, Las Palmas de Gran Canaria, Spain, 2004.

(21) Zienkiewicz, O. C.; Taylor, R. The method of finite elements; McGraw-Hill: New York, 1995.

(22) Dular, P.; Geuzaine, C. GetDP: A general environment for the treatment of discrete problems. http://geuz.org/getdp/ (accessed 06/06/06). 
(23) Janaun, A. Numerical methods for partial differential equations; Royal Institute of Technology of Stockholm: Stockholm, Sweden, 2003.

(24) Driesen, J. Coupled Electromagnetic-Thermal Problems in Electrical Energy Transducers. Ph.D. Thesis, Katholieke Universiteit Leuven, Herverlee, Belgium, 2000.

(25) Geuzaine, C.; Remacle, J. F. Gmush: a three-dimensional finite element mesh generator with built-in -pre-and postprocessing facilities. http://geuz.org/gmsh/ (accessed 06/06/06).

(26) Pendse, H.; Tien, C. General correlation of the initial collection efficiency of granular filter beds. AIChE J. 1982, 28, 677.

(27) Ergun, S. Fluid flow through packed columns. Chem. Eng. Prog. 1952, 48,89 .

(28) Hinds, W.; Kadrichu, N. P. The effect of dust loading on penetration and resistance of glass fiber filters. Aerosol Sci. Technol. 1997, 27, 162.

(29) Brown, R. C. Air filtration: An integrated approach of the theory and applications of fibrous filters; Pergamon: Oxford, U.K., 1993.

(30) Kanaoka, C.; Hiragi, S. Pressure drop of air filter with dust load. J. Aerosol Sci. 1990, 21, 127.

(31) Chiang, H. W.; Tien, C. Dynamic of the deep bed filtration. Part. 1: Analysis of two limiting solutions. AIChE J. 1985, 31, 1349.

(32) Stamakis, K.; Tien, C. A simple model of cross-flow filtration based on particle adhesion. AIChE J. 1993, 31, 1292.

(33) Peukert, W.; Löfler, F. Influence of temperature on particle separation in granular bed filters. Powder Technol. 1991, 68, 263.
(34) Stovall, T.; De Larrard, F.; Buil, M. Linear parking density of grain mixtures. Powder Technol. 1986, 48, 1-12.

(35) Yu, A. B.; Standish, N. Estimation of the porosity of particles mixtures by a linear-mixture packing model. Ind. Eng. Chem. Res. 1991, $30,1372$.

(36) Ouchiyama, N.; Tanaka, T. Predicting the densest packing of ternary and quaternary mixtures of solid particles. Ind. Eng. Chem. Res. 1989, 28, 1530 .

(37) Yu, A. B.; Standish, N. Limitation of proposed mathematical model for the porosity estimation of nonspherical particle mixtures. Ind. Eng. Chem. Res. 1993, 32, 2179.

(38) Yung, Y.; Tien, C. Increase in collector efficiency due to deposition in polydispersed granular filtration-An experimental study. J. Aerosol Sci. 1992, 23, 525.

(39) Levenspiel, O. Engineering flow and heat exchange; Plenum Press: New York, 1984.

Received for review March 23, 2006 Revised manuscript received August 24, 2006 Accepted August 28, 2006

IE060354K 